INPLASY

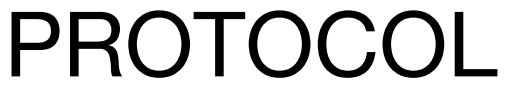

To cite: Guede-Rojas et al. Effectiveness of exergames on proprioception in older adults. A systematic review protocol. Inplasy protocol 202170060. doi:

10.37766/inplasy2021.7.0060

Received: 19 July 2021

Published: 19 July 2021

Corresponding author:

Francisco Guede-Rojas

francisco.guede@unab.cl

Author Affiliation:

Universidad Andres Bello

Support: None.

Review Stage at time of this submission: Preliminary searches.

Conflicts of interest:

None declared.

\section{Effectiveness of exergames on proprioception in older adults. A systematic review protocol}

Guede-Rojas, F1; Cáceres-Vejar, B2; Godoy-Escobar, M33; Tripaili-Fernández, A4; Vera-Nanjari, C $^{5}$.

Review question / Objective: In older adults with or without specific clinical conditions, are exergames based on nonimmersive virtual reality systems effective on proprioception? Condition being studied: Aging corresponds to a physiological process that is expressed throughout the entire life cycle, being determined by the progressive decrease in multisystemic homeostatic reserves that affect functional capacity in older people. Proprioception is defined as the sensation of joint movements, as well as the position of the body segments in space. Proprioceptive information is determined by elements of the nervous system such as the sensory system (vestibular, visual, and somatosensory) that provides information on the location and orientation of movements; the supraspinal centers in charge of planning, initiating and executing these movements; and the subcortical centers (spinal cord and brainstem) that guarantee adequate postural adjustments. Therefore, in older adults, proprioception takes great importance on motor control, gait, maintenance of balance and posture. There are reports of complementary therapies on proprioception in older adults such as Tai-chi, Yoga and Pilates. On the other hand, programs based on proprioceptive training have been developed on the improvement of various components of functional physical performance in older people.

INPLASY registration number: This protocol was registered with the International Platform of Registered Systematic Review and Meta-Analysis Protocols (INPLASY) on 19 July 2021 and was last updated on 19 July 2021 (registration number INPLASY202170060).

\section{INTRODUCTION}

Review question / Objective: In older adults with or without specific clinical conditions, are exergames based on non-immersive virtual reality systems effective on proprioception? 
Rationale: Virtual reality (VR) refers to an interactive simulated digital environment that gives the user the perception of being physically in the game. VR is classified as: immersive, semi-immersive, and nonimmersive. The latter modality corresponds to the most accessible and with the lowest level of immersion, where users interact through screens or monitors linked to video game consoles or motion capture systems. Exergames correspond to any type of video game that requires active movements controlled by the player. Among its advantages, the development of motor and cognitive skills, as well as its easy adaptation. Overall, training with "non-immersive VR" in older adults has proven to be feasible, acceptable and safe. This has been confirmed through rates of attendance, dropout and self-perception questionnaires. Finally, these systems are easy to use and well accepted among the elderly as shown through qualitative and quantitative methods. The effectiveness of exergames on various functional performance outcomes in older adults has been analyzed through systematic reviews in the field of neurorehabilitation (stroke, traumatic brain injury, and Parkinson's disease, a mong others) and musculoskeletal (chronic pain, and hip/ knee osteoarthritis), as well as in healthy community-dwelling older adults. However, to the best of the authors' knowledge, no systematic review has analyzed the effectiveness of exergames on proprioception in older adults.

Condition being studied: Aging corresponds to a physiological process that is expressed throughout the entire life cycle, being determined by the progressive decrease in multisystemic homeostatic reserves that affect functional capacity in older people. Proprioception is defined as the sensation of joint movements, as well as the position of the body segments in space. Proprioceptive information is determined by elements of the nervous system such as the sensory system (vestibular, visual, and somatosensory) that provides information on the location and orientation of movements; the supraspinal centers in charge of planning, initiating and executing these movements; and the subcortical centers (spinal cord and brainstem) that guarantee adequate postural adjustments. Therefore, in older adults, proprioception takes great importance on motor control, gait, maintenance of balance and posture. There are reports of complementary therapies on proprioception in older adults such as Taichi, Yoga and Pilates. On the other hand, programs based on proprioceptive training have been developed on the improvement of various components of functional physical performance in older people.

\section{METHODS}

Search strategy: (1) Population: (()( ( ( ( ( ( ( geriatrics [MeSH Terms]) OR (aged[MeSH Terms])) OR (aging[MeSH Terms])) OR (frail elderly[MeSH Terms])) OR (geriatr*)) OR (gerontol*)) OR (ageing)) OR (elder*)) OR ("older adult*")) OR ("older people")) OR ("older person*")) OR ("old age")) OR (Senior*) (2) Intervention:

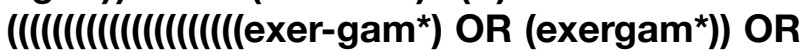
(video games[MeSH Terms])) OR (videogam*)) OR (video-gam*)) OR (videobased)) OR ("gaming technology")) OR (wii)) OR (nintendo)) OR (kinect)) OR (xbox)) OR (x-box)) OR (eyetoy)) OR ("Play station")) OR (Play-station)) OR ("Serious gam*")) OR ("Dance dance revolution")) OR ("active video gam*")) OR (Non-immersive)) OR ("Non immersive")) OR (Nonimmersive)) OR (virtual reality[MeSH Terms]) (3) Outcomes: (()((((Proprioception[MeSH Terms]) OR $($ propriocep*)) OR (kinesthesis [MeSH Terms])) OR (kinesthe*)) OR (kinaesthe*)) OR ("position sense")) OR ("joint position sense")) OR ("sense of resistance").

Participant or population: Older adults.

Intervention: Exergames based on nonimmersive virtual reality.

Comparator: Control group: No intervention, conventional physical therapy, or other therapeutic modalities.

Study designs to be included: Randomized controlled trials (RCTs). 
Eligibility criteria: (1) Inclusion criteria: Population: Older adults of both sexes, older than 60 years. With or without pathology or clinical conditions; Intervention: Exergames performed through any system or console based on non-immersive virtual reality; Comparator: Control group: No intervention, conventional physical therapy, or other therapeutic modalities; Outcomes: Proprioception measures. No restriction of specific joints or measuring instruments; Time: Studies of the last 10 years due to the rapid development of new technologies and the need to obtain the most current results; Type of study: Randomized controlled trials (RCTs); Setting: no restriction; Language: English. (2) Exclusion criteria: Unpublished studies, clinical trial protocols, secondary studies, and conference reports.

Information sources: Electronic searches will be carried out in the databases: Scopus, Web of Science (WOS) and PubMed. The electronic search will be complemented by a manual search from the reference list of the included studies.

Main outcome(s): Any specific proprioception measurements carried out using laboratory instruments or clinical tests. The outcome indicators are continuous variables. Measures of effect: Difference of means (MD) or standard difference of means (SMD).

\section{Additional outcome(s): None.}

Data management: (1) The results of the databases will be imported to the Rayyan platform. (2) After eliminating duplicates, two independent reviewers will manage the selection of studies. The concordance will be analysed using the kappa index. (3) The selection process will initially consider a screening for titles and abstract, and then a comprehensive review of the full text. (4) Reviewers will independently extract information from included studies. (5) The data extraction form will include: Author; Year of publication; Primary objective; Setting; Population (sample size, distribution by sex, mean age, clinical condition, inclusion criteria); Intervention (experimental - control groups, technology / systems used, frequency of intervention (number of weeks and sessions / week); Results measurement tools; Main results; Measures of effect magnitude or clinical significance; Background related to: Adverse events, feasibility and usability. (6) Possible conflicts at each stage of study selection and data extraction will be resolved by consensus.

Quality assessment / Risk of bias analysis: (1) The 11-point PEDro scale. For the assessment of the methodological quality of individual studies. (2) Cochrane Revman 5.4 Software. For the assessment of individual and overall risk of bias of the studies.

Strategy of data synthesis: According to the number of included studies their comparisons and their heterogeneity, the possibility of performing a meta-analysis using the Revman 5.4 software will be evaluated. We will decide to use a fixed effects model if heterogeneity is low $\left(I^{2}<40 \%\right)$ or a random effects model assuming a more conservative estimate of the real effect if heterogeneity is higher $\left(I^{2}\right.$ $40 \%$ and $<75 \%$ ). Both models involve weighting the estimate of the effect size by the inverse of the variance. Heterogeneity will be calculated using $I^{2}(0 \%$ to $40 \%$ might not be important; $30 \%$ to $60 \%$ may represent moderate heterogeneity; $50 \%$ to $90 \%$ may represent substantial heterogeneity; $75 \%$ to $100 \%$ considerable heterogeneity. The Chi-square test will be used to evaluate whether the differences observed in the results were compatible with chance (p75\%), a qualitative synthesis of the findings will be performed.

Subgroup analysis: A possible subgroup analysis may be carried out depending on the clinical characteristics of the participants, the types of intervention or the proprioception measurement methods.

Sensitivity analysis: To analyze the stability of the conclusions, studies with lower 
methodological quality and higher risk of bias will be excluded.

Language: English only.

Country(ies) involved: Chile.

Other relevant information: None.

Keywords: Geriatrics; Aging; Video games; Virtual reality; Proprioception; Kinesthesis.

Dissemination plans: Peer-reviewed indexed journal.

Contributions of each author:

Author 1 - Francisco Guede-Rojas - Study design, guidance and drafted the manuscript.

Email: francisco.guede@unab.cl

Author 2 - Bárbara Cáceres-Vejar Literature search and selection.

Email: barbicaceresj@gmail.com

Author 3 - Melanie Godoy-Escobar Literature search and selection.

Email: godoymelanie98@gmail.com

Author 4 - Aracely Tripaili-Fernández - Data collection and synthesis.

Email: Aatf121@gmail.com

Author 5 - Camila Vera-Nanjari - Data collection and synthesis.

Email: camivera13@gmail.com 